\title{
Self-Excited Vibration of Propeller Nozzles Experienced on Ocean-Going Tugboats
}

\author{
-1st Report- \\ by Yasunosuke Ogawa*, Member Shinji Kumazaki*, Member \\ Makoto Maeda*, Member Katsuya Fujii**, Member \\ Isao Neki*, Member Masatetsu Shimono* \\ Yasuhisa Okumoto*, Member
}

\begin{abstract}
Summary
Despite every means available at the time of construction applied to prevent vibration, violent vibrations were reported on two ocean-going tugboats constructed by IHI, of size that places them among the world's largest. Measurements made of the vibration of aft part including nozzles and analysis of measured data revealed that the vibrations of nozzles:-

-were constant in frequency, irrespective of propeller or engine revolution, and attained values of acceleration reaching $8,000 \mathrm{Gal}$;

-were generated only under certain conditions of operation, and diminished to very low levels under all other conditions;

-corresponded to what is known as flow-induced vibration, and were closely associated with the water flow around the nozzles.

Two possibly ascribable mechanisms were considered as cause of the self-excited vibration.

(1) Locked-in vibration induced by Karman's vortex street;

(2) Occurrence of negative damping (known as "galloping").

Neither of these two mechanisms have so far proved conclusively assignable as cause: (1) Karman's vortex streets on account of uncertainty in estimating the exciting force under lock-in condition, and (2) galloping for lack of definite tendency of the negative slope of nozzle lift vs. attack angle curve.

The vibrations observed being of such amplitude as to permit no delay in remedial action, measures were taken to counteract both the mechanisms considered above: (1) Karman's vortex streets by attaching fins to the nozzles to decrease their trailing edge thickness, and hence to modify the shedding frequency of the vortex streets, and avoid resonance; (2) galloping by strutting the two nozzles together so as to constrain them to in-phase vibration mode, which was considered from observation of the damping behavior to be conductive to marked modification of the structural and mass damping performance, and consequently convert the damping to positive value.

Vibration measurements reperformed after applying the above measures proved the violent vibrations to have been completely eliminated, evidencing ample effectiveness of the measures adopted. Further token of the effectiveness of the measures is provided by the complete absence of reports from either tugboat on violent vibrations, throughout the two years that have since elapsed, during which the tugboats have each marked over 4,000 hours of active service.
\end{abstract}

* Shipbuilding \& Offshore, IshikawajimaHarima Heavy Industries Co., Ltd.

** Yokohama Research Institute, Ishikawajima-Harima Heavy Industries Co., Ltd.

\section{Introduction}

Two ocean-going tugboats-of capacity that places them amongst the world's largest-were completed by IHI in October and December 1979. At the time of their construction, every means 
currently available was applied to prevent vibration $^{1)}$, but nevertheless, after their delivery, the crew of both tugboats reported violent vibrations experienced in the accommodation space and the aft part where the propellers and nozzles were equiped. In January 1982, an extensive series of vibration measurements was carried out jointly between IHI and the Owners of the tugboats. The measurements were made with underwater accelerometers mounted on the nozzles and surrounding parts.

It was established as a result that the vibrations occurring on the nozzles and around stern.

-were constant in frequency, irrespective of propeller or engine revolution, and attained values of vibration acceleration reaching $8,000 \mathrm{Gal}$,

-were generated only under certain conditions of operation, and diminished to very low levels under all other conditions,

-corresponded to what is known as flowinduced vibration, and were closely associated with the water flow around the nozzle. The foregoing factors were indicative of a phenomenon that was hardly foreseeable from the state of knowledge of ship vibrations at the time of construction of the tugboats.

Based on the information thus assembled, together with the data that had been recorded at the time of construction, measures for eliminating the violent vibrations were planned, and were applied to the tugboats in April and September 1982. Vibration measurements were then remade, to verify the effectiveness of the measures thus applied.

Two years have elapsed since then, and the tugboats have each already marked over 4,000 hours of active service, but no reports have since been received of violent vibrations experienced in either tugboat.

In this paper, the characteristics of the violent vibrations are noted, their probable causes are discussed, the measures successfully applied are indicated, and the effectiveness of the measures are verified.

\section{Principal Particulars of the Tugboats}

The rough arrangement of the tugboats is shown in Fig. 1; Table 1 presents the principal particulars. Notable characteristics are: Twinengine, twin-screw, controllable-pitch propellers rotating in fixed nozzles; 20,800 SHP engine output, $200 \mathrm{tf}$ towing force, $19.4 \mathrm{kt}$ service speed.

As shown in Fig. 2, the $4 \mathrm{~m}$ diameter nozzle is connected above through head box to the stern, and below to the lower end of the shaft bracket by means of strut. This arrangement of nozzle support was adopted on considerations detailed in
Table 1 Principal particulars

\begin{tabular}{|l|r|}
\hline$L b p$ & $90.0 \mathrm{~m}$ \\
\hline Molded breadth & $15.8 \mathrm{~m}$ \\
\hline Molded depth & $8.0 \mathrm{~m}$ \\
\hline Molded draft & $6.0 \mathrm{~m}$ \\
\hline Main engine & $\begin{array}{c}\text { IHI-S.E.M.T.Pielstick } \\
\text { diesel engine } 16 \mathrm{PC2}- \\
5 \mathrm{~V} \times 2 \text { sets }\end{array}$ \\
\hline $\begin{array}{l}\text { Maximum output } \\
\text { of main engine }\end{array}$ & $\begin{array}{l}10,400 \text { PS } \times 520 \mathrm{rpm} \\
\times 2 \text { sets }\end{array}$ \\
\hline $\begin{array}{l}\text { Propeller } \\
\text { 4-blade controllable } \\
\text { pitch propeller } \\
\text { (with fixed nozzle) } \\
\times 2 \text { sets }\end{array}$ \\
\hline $\begin{array}{l}\text { Revolutions (main } \\
\text { engine/propeller) }\end{array}$ & $340-520 / 110-168 \mathrm{rpm}$ \\
\hline $\begin{array}{l}\text { Speed } \\
\text { Maximum towing } \\
\text { force }\end{array}$ & $\begin{array}{l}19.4 \mathrm{kt} \\
\text { Classification }\end{array}$ \\
\hline
\end{tabular}
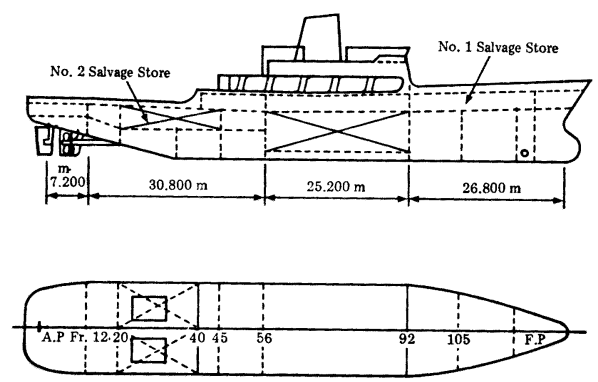

Fig. 1 Rough arrangement

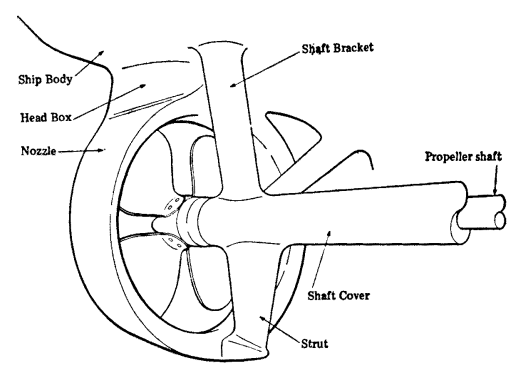

Fig. 2 Plan of nozzle

Reference ${ }^{1)}$.

\section{Vibration Measurements}

\subsection{Arrangement for measurements}

While the tugboats were docked, underwater accelerometers were mounted on the nozzles and surrounding parts protected by watertight sealing, as indicated in Fig. 3, and vibration measurements were made after undocking.

The conditions at measurement are presented in Table 2. Runs 1 and 3 were made with tugboat running free, of which Run 1 was made under normal conditions of auto-program control, in which tugboat speed was controlled by propeller 
Table 2 Ship's condition

\begin{tabular}{|c|c|c|c|c|}
\hline NO. & 1 & 2 & 3 & 4 \\
\hline ITEM & $\begin{array}{c}\text { PROGRESSIVE } \\
\text { SHAFT R.P.M. } \\
\text { MEASUREMENT }\end{array}$ & $\begin{array}{c}\text { PRELIMINARY } \\
\text { TOWING } \\
\text { MEASUREMENT }\end{array}$ & $\begin{array}{c}\text { PROGRESSIVE } \\
\text { BLADE PITCH } \\
\text { MEASUREMENT }\end{array}$ & $\begin{array}{c}\text { TOWING } \\
\text { MEASUREMENT }\end{array}$ \\
\hline DATE & 16 TH JAN '82 & 24.TH JAN '82 & 24TH JAN '82 & 28TH JAN '82 \\
\hline PLACE & OFF EJMMA & OSAKA BAY & OSAKA BAY & OSAKA BAY \\
\hline WEATHER & FINE & FINE & FINE & CLOUDY \\
\hline $\begin{array}{c}\text { SEA } \\
\text { CONDITION }\end{array}$ & CALM & CALM & CALM & SLIGHT \\
\hline $\begin{array}{c}\text { DEPTH OF } \\
\text { WATER }\end{array}$ & $\begin{array}{c}\text { DEEPER THAN } \\
20 \mathrm{~m}\end{array}$ & $\begin{array}{c}\text { DEEPER THAN } \\
16 \mathrm{~m}\end{array}$ & $\begin{array}{c}\text { DEEPER THAN } \\
16 \mathrm{~m}\end{array}$ & $\begin{array}{c}\text { DEEPER THAN } \\
16 \mathrm{~m}\end{array}$ \\
\hline $\begin{array}{c}\text { DISPLACE- } \\
\text { MENT }\end{array}$ & abt 4,700 $\mathrm{t}$ & abt $4,800 \mathrm{t}$ & abt $4,800 \mathrm{t}$ & abt $4,900 \mathrm{t}$ \\
\hline$d f$ & $5.3 \mathrm{~m}$ & $5.3 \mathrm{~m}$ & $5.3 \mathrm{~m}$ & $5.3 \mathrm{~m}$ \\
\hline$d a$ & $5.5 \mathrm{~m}$ & $5.7 \mathrm{~m}$ & $5.7 \mathrm{~m}$ & $5.8 \mathrm{~m}$ \\
\hline$d m$ & $5.4 \mathrm{~m}$ & $5.5 \mathrm{~m}$ & $5.5 \mathrm{~m}$ & $5.55 \mathrm{~m}$ \\
\hline $\begin{array}{c}\text { PROPELLER } \\
\text { IMMERSION }\end{array}$ & $120 \%$ & $125 \%$ & $125 \%$ & $128 \%$ \\
\hline $\begin{array}{c}\text { NOZZLE } \\
\text { MMMERSION }\end{array}$ & $107 \%$ & $111 \%$ & $111 \%$. & $113 \%$ \\
\hline $\begin{array}{c}\text { TOWING } \\
\text { FORCE }\end{array}$ & $0 \mathrm{t}$ & max. $45 \mathrm{t}$ & $0 \mathrm{t}$ & max. $153 \mathrm{t}$ \\
\hline
\end{tabular}
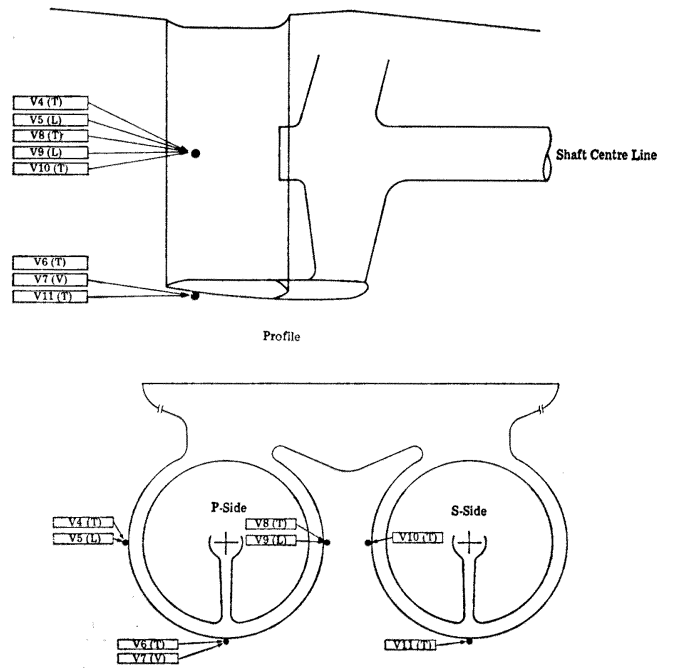

Fig. 3 Arrangement of measuring points

blade pitch at $110 \mathrm{rpm}$ propeller revolution, and above this revolution, controlled by propeller revolution with blade pitch fixed at about $32^{\circ}$. Run 3 was made with propeller revolution held constant and with tugboat speed controlled by propeller blade pitch. Runs 2 and 4 were made while maximum towing loads of 45 and $153 \mathrm{tf}$, respectively.

The measured revolutions and propeller pitch angles are indicated in Fig. 4.

\subsection{Results of measurement}

\subsubsection{Run 1 (auto-program control)}

Measurements were made with propeller revolution varied from 110 to $163 \mathrm{rpm}$ in steps of 3 $\mathrm{rpm}$. The strongest vibration was recognized at the measuring point V6, the frequencies composing the vibration at this point being as represented by the spectrum of Fig. 5. The accelera-

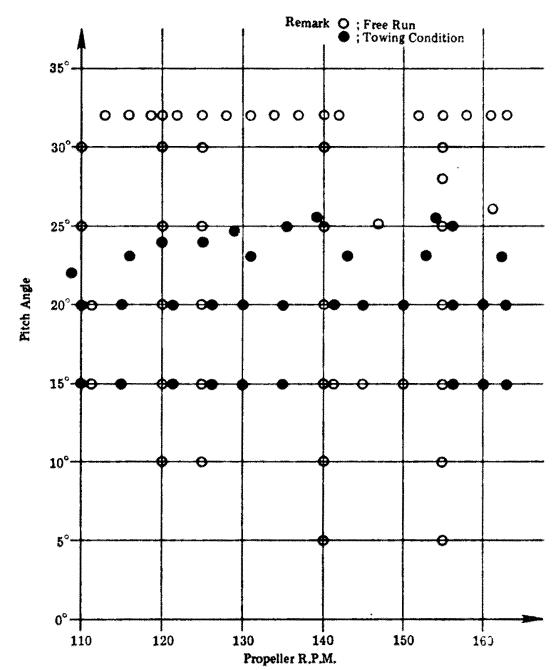

Fig. 4 Measuring pitch angle and propeller RPM

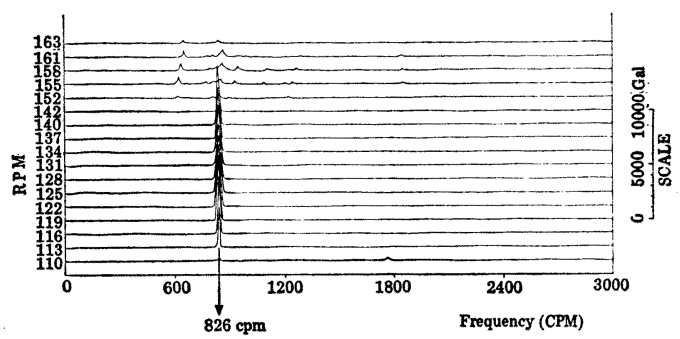

Fig. 5 Spectrum analysis V6(T)

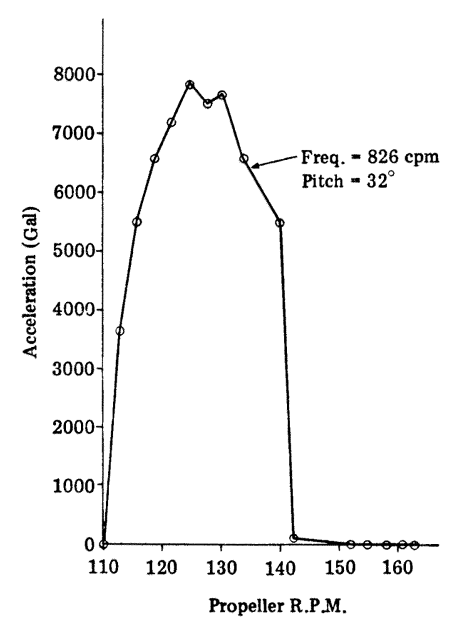

Fig. 6 Vibration acceleration V6(T)

tion level at $826 \mathrm{cpm}$ for each propeller revolutions is shown in Fig. 6; Fig. 7 represents the vibration mode measured at $125 \mathrm{rpm}$, at which the highest acceleration was recognized. During these 


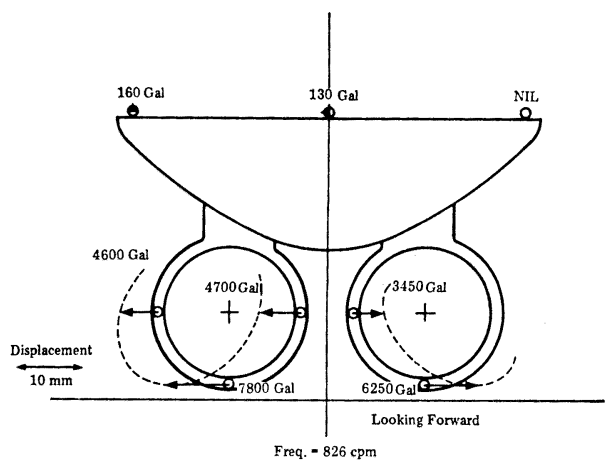

Fig. 7 Vibration mode (125 RPM) pitch $=$ $32^{\circ}$

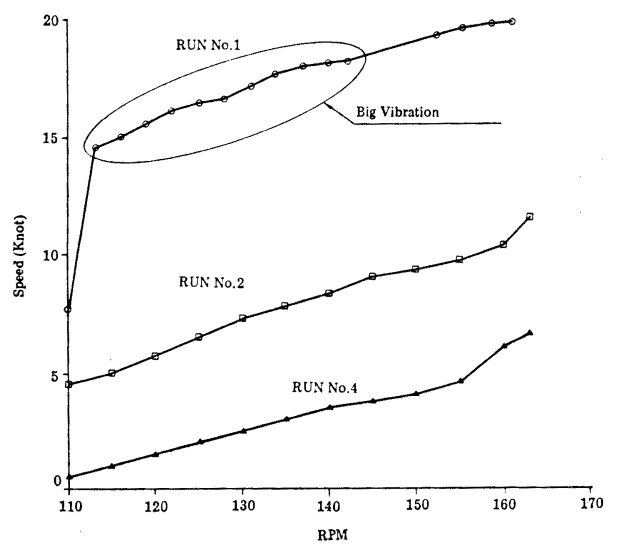

Fig. 8 Ship's speed at vibration measurements

measurements, the tugboat speed changed as shown in Fig. 8.

The foregoing data indicate that:

(1) Vibration was prominent in the range of revolutions from 113 to $140 \mathrm{rpm}$; outside this range, the vibrations diminished markedly.

(2) The violent vibration was generated uniquely at the constant frequency of $826 \mathrm{cpm}$.

(3) The nozzles vibrated laterally in anti-phase mode; vibration was slightly higher level on the port side nozzle.

(4) The violent vibration was generated at 14 to $18 \mathrm{kt}$ tug speed.

\subsubsection{Run 3 (pitch controll)}

This run was made to examine the relation between propeller blade pitch and vibration. The accelerometer readings from the measuring point V6 at 125 and $140 \mathrm{rpm}$ are reproduced in Fig. 9, where the time scale is reduced for clarity.

It is seen that vibration is negligible when the propeller blade pitch is below $30^{\circ}$, beyond which
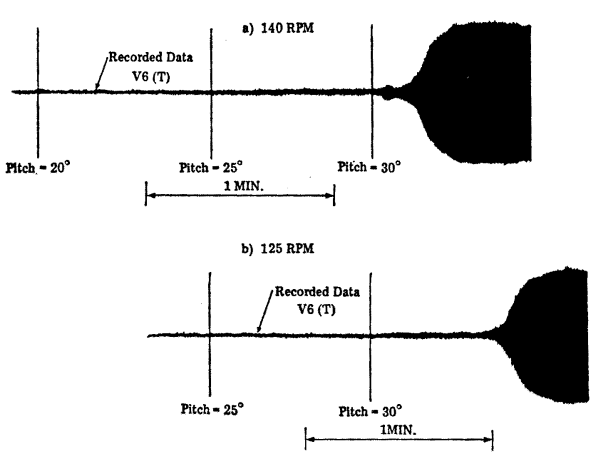

Fig. 9 Effect of pitch

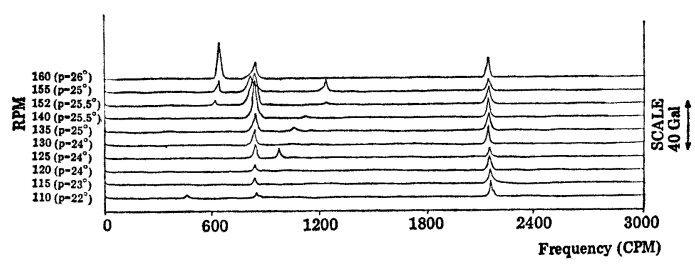

Fig. 10 Spectrum analysis V6(T) (towing condition)

it increases sharply.

\subsubsection{Runs 2 and 4 (towing condition)}

An example of vibration analysis is presented in Fig. 10, which reveals that when towing, the violent vibrations observed when running free were no longer generated, the highest acceleration recorded being only $32 \mathrm{Gal}$ at measuring point V6 in both Runs.

\section{Discussion on Cause of Vibration}

A discussion is presented on the probable mechanism that generates the extraordinary vibrations noted in the preceding Section 3.2.

\subsection{Measured vibration magnitude}

Fig. 11 presents the relation between propeller RPM and frequencies of the measured data given in Fig. 5.

It is revealed that the violent vibrations correspond to none of the frequencies that would normally be considered to affect the stern part of hull-i.e. multiples of propeller shaft revolution (1, $4=$ Number of blades, $8=$ Twice number of blades, $\cdots$ - -and that the frequency is constant and irrespective of propeller revolution. This frequency of $826 \mathrm{cpm}$ is, as shown in Fig. 12, close to the natural frequency of $800 \mathrm{cpm}$ measured with vibration exciter applied to hull stern while moored $^{1)}$, and the present value can be considered to represent the corresponding natural frequency while running.

The above behavior of the inordinate vibra- 


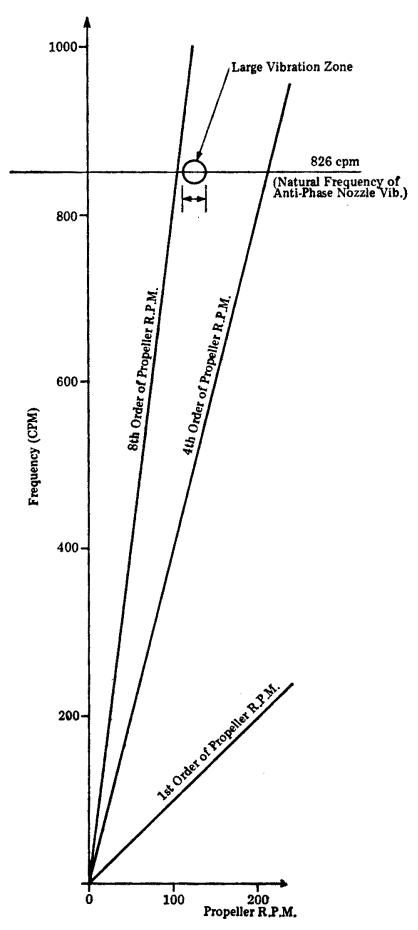

Fig. 11 Relation between propeller RPM and frequencies
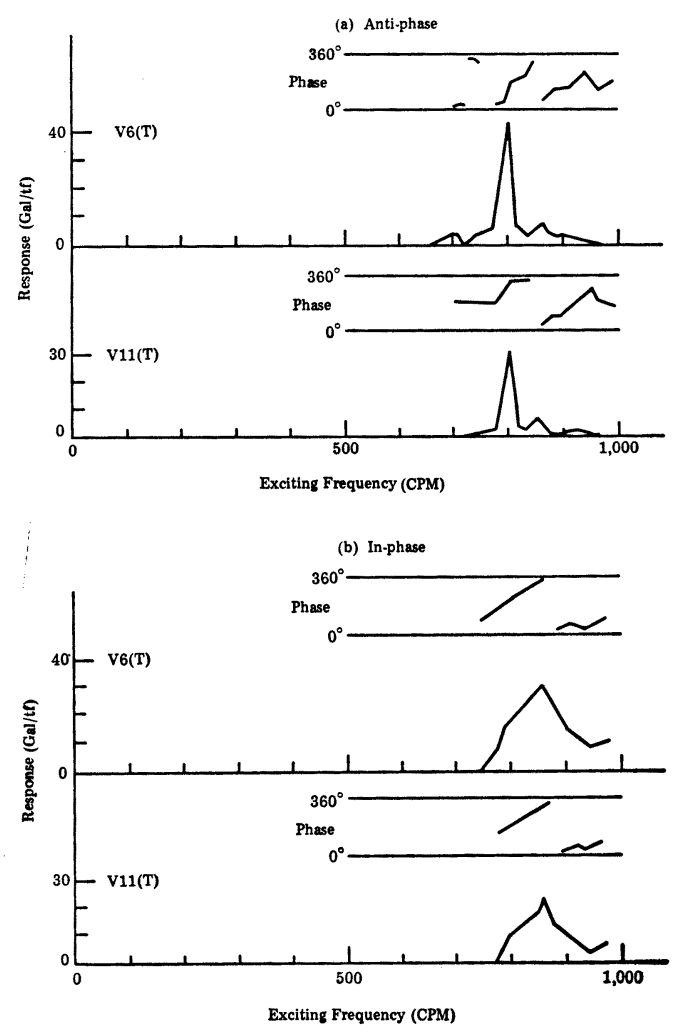

Fig. 12 Results of exciter test tions is characteristic of what is known as self. excited vibration.

\subsection{Self-excited vibration}

Upon examining the various factors that govern the self-excited vibration affecting the nozzles, the probable causes were narrowed down to:

(1) Locked-in vibration caused by Karman's vortex street

(2) Self-excited vibration generated on account of the nozzle damping characteristics becoming negative-i.e. phenomenon known as "galloping".

\subsubsection{Karman's vortex street}

We first examined the likelihood of the cause of violent vibrations being Karman's vortex street, which is rarely experienced in practice.

The exciting frequency should in such case be

$$
f=60 \cdot U_{0} \cdot c / d, \quad(\mathrm{cpm})
$$

where

$U_{0}:$ Flow velocity $(\mathrm{m} / \mathrm{s})$

$d$ : Nozzle trailing edge thickness $(=0.1 \mathrm{~m})$

$c$ : Strouhal coefficient $(=0.15-0.20)$

Substituting into Eq. (4.1) the tugboat speed derived in Section 3.2.1 (4), the estimated exciting frequency

$$
f=648-1,110 \mathrm{cpm},
$$

is obtained.

The frequency of $826 \mathrm{cpm}$ obtained from actual measurement is situated in the mid range of this exciting frequency. This suggests the violent vibrations in question to be assignable to what is known as forced self-excited vibration by Karman's vortex street with the vortex-shedding frequency in the above range locked-in to the 826 cpm of nozzle natural frequency.

The exciting force can be estimated practically i as follows ${ }^{2)}$,

$$
w=1 / 2 \cdot \rho U_{0}^{2} d \mathrm{C}_{L} Q,
$$

where

$w$ : Exciting force per unit length of nozzle $(\mathrm{kgf} / \mathrm{m})$

$\rho$ : Specific gravity of fluid $\left(=104.6 \mathrm{kgf} \cdot \mathrm{s}^{2} /\right.$ $\left.\mathrm{m}^{4}\right)$

$C_{L}$ : Lift coefficient for round $\mathrm{bar}^{2)}(=0.15)$

$Q$ : Magnification factor $(=80)$.

Therefore, in the flow velocity range of 14 to 18 $\mathrm{kt}$ which corresponds to the violent vibrations,

$$
w=3255-5382 \mathrm{kgf} / \mathrm{m} \text {. }
$$

If the vortex street exciting force is considered to have been generated in the part indicated in Fig. 13 , the total exciting force

$$
F=w \cdot \pi \cdot D \cdot 120 / 360 \mathrm{kgf},
$$




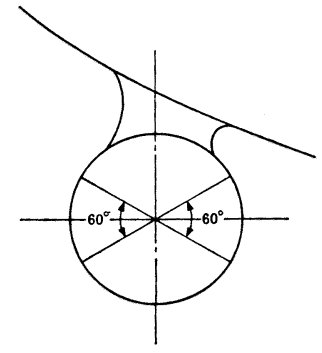

Fig. 13 Assumed range of Karman's vortex street

where

$D:$ Nozzle diameter $(=4 \mathrm{~m})$.

Therefore,

$$
F=13,635-22,544 \mathrm{kgf} \text {. }
$$

The spring constant of the nozzle structure is calculated as $180 \mathrm{tf} / \mathrm{mm}$, as derived from its transverse rigidity which had been determined at the time of initial design. Hence, the amplitude of vibration that would be excited by the Karman's vortex street would be

$$
\begin{aligned}
\delta & =F / 180,000 \\
& =0.076-0.125 \mathrm{~mm},
\end{aligned}
$$

which is 2 orders of magnitude smaller than the measured value of around $10 \mathrm{~mm}$ and this is one element of doubt on the cause by Karman's vortex street. Another element of doubt is whether the vortex street would actually exert a force conductive to lateral vibration of the nozzles, when continuity of the vortexes is considered.

On the other hand, it has been found ${ }^{3)}$ that once Karman's vortex street would be lockedin, the value of $C_{L}$ in Eq. (4.2) can depart widely from that of forced vibration, and the results, the vibration amplitude can be extraordinary increased.

The effect of this lock-in would not permit Karman's vortex street to be immediately dismissed as possible cause of the violent vibrations.

\subsubsection{Self-excitation by negative damping}

(1) Mechanism

A freely damping system of 1 degree of freedom will be governed by the equation of motion

$$
\ddot{y}+2 \varepsilon \dot{y}+\nu^{2} y=0,
$$

which represents a periodic motion when $\varepsilon<\nu$, and a general solution can be obtained as

$$
y=A e^{-\varepsilon t} \cdot \cos \left(\sqrt{\nu^{2}-\varepsilon^{2}} \cdot t+B\right),
$$

where, letting the period $T=2 \pi / \sqrt{\nu^{2}-\varepsilon^{2}}$, the ratio of amplitudes between the instants $t_{0}$ and $t_{0}+T$ should become

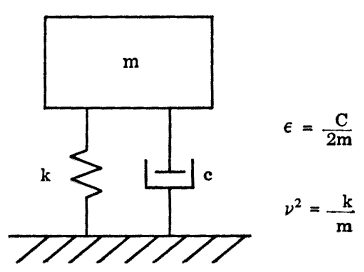

Fig. 14 Model of free vibration

$$
\left|y_{t=t_{0}+T}\right| /\left|y_{t=t_{0}}\right|=e^{-\varepsilon T}
$$

which indicates a vibration attenuating with each cycle if $\varepsilon>0$, whereas when $\varepsilon<0$ an amplifying vibration that strengthens with each cycle is indicated.

The latter case represents the mechanism of self-excitation generated by negative damping.

(2) Application to the present instance

The nozzle placed in fluid stream, modelized for simplicity to represent one degree of freedom, is illustrated in Fig. 15, in which diagram,

$$
\begin{aligned}
& F_{L}=1 / 2 \rho U_{R}^{2} D^{2} C_{L} \\
& F_{D}=1 / 2 \rho U_{R}^{2} D^{2} C_{D},
\end{aligned}
$$

where

$F_{L}:$ Lift for one nozzle king $(\mathrm{kg})$

$F_{D}$ : Drag for one nozzle ring $(\mathrm{kg})$

$U_{R}:$ Resultant velocity $(\mathrm{m} / \mathrm{s})$

$C_{L}$ : Lift coefficient of one nozzle ring

$C_{D}$ : Drag coefficient of one nozzle ring

We here assume that:

$$
\begin{aligned}
& \dot{x}=0, U_{x}=U_{0} ; \\
& U_{y}=0,|\dot{y}| \ll U_{0}, \alpha=-\dot{y} / U_{0} ; \\
& U_{R}^{2}=U_{0}^{2}+\dot{y}^{2} .
\end{aligned}
$$

Now, the resultant force in the $y$-direction

$$
\begin{aligned}
F_{y}= & F_{L} \cos \alpha+F_{D} \sin \alpha \\
& =1 / 2 \rho U_{R}{ }^{2} D^{2}\left(C_{L} \cos \alpha+C_{D} \sin \alpha\right) .
\end{aligned}
$$

Hence, the equation of motion governing the model of Fig. 15 becomes

$$
\left(m+m_{\mathrm{add}}\right) \ddot{y}+C \dot{y}+k y=F_{y},
$$

where

$$
m_{\text {add }}: \text { Added water mass. }
$$

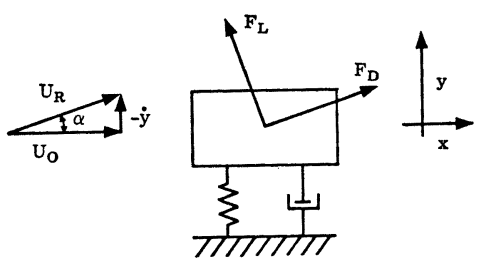

Fig. 15 Model of nozzle in flow 
By letting

$$
F_{y}=1 / 2 \rho U_{0}^{2} D^{2} C_{y},
$$

and substituting this $F_{y}$ into Eq. (4.8), we have

$$
C_{y}=\left(C_{L} \cos \alpha+C_{D} \sin \alpha\right)\left(U_{R} / U_{0}\right)^{2} .
$$

On the other hand, since $\alpha$ is very small,

$$
\begin{aligned}
& C_{L}(\alpha)=C_{L}(0)+\left(\frac{\partial C_{L}}{\partial \alpha}\right)_{\alpha=0} \cdot \alpha+0\left(\alpha^{2}\right) \\
& C_{D}(\alpha)=C_{D}(0)+\left(\frac{\partial C_{D}}{\partial \alpha}\right)_{\alpha=0} \cdot \alpha+0\left(\alpha^{2}\right) \\
& \cos \alpha=1-0\left(\alpha^{2}\right) \\
& \sin \alpha=\alpha \\
& \left(U_{R} / U_{0}\right)^{2}=1+0\left(\alpha^{2}\right) .
\end{aligned}
$$

By substituting Eq. (4.12) into Eq. (4.11),

$$
C_{y}=C_{L}(0)-\left\{\left(\frac{\partial C_{L}}{\partial \alpha}\right)_{\alpha=0}+C_{D}(0)\right\}\left(\dot{y} / U_{0}\right) .
$$

In this equation, $C_{L}(0)$ is a static load, so that the dynamic component

$$
C_{y}=-\left\{\left(\frac{\partial C_{L}}{\partial \alpha}\right)_{\alpha=0}+C_{D}(0)\right\}\left(\dot{y} / U_{0}\right) .
$$

By substituting Eqs. (4.14), (4.10) into Eq. (4.9), we derive the equation of motion

$$
\begin{gathered}
\left(m+m_{\text {add }}\right) \ddot{y}+\left[C+(1 / 2) \rho U_{0} D^{2}\left\{\left(\frac{\partial C_{L}}{\partial \alpha}\right)_{\alpha=0}\right.\right. \\
\left.\left.+C_{D}(0)\right\}\right] \dot{y}+k \ddot{y}=0,
\end{gathered}
$$

where

$$
\begin{gathered}
C \text { : Structural and mass damping } \\
1 / 2 \rho U_{0} D^{2}\left\{\left(\frac{\partial C_{L}}{\partial \alpha}\right)_{\alpha=0}+C_{D}(0)\right\}: \\
\text { Dynamic damping. }
\end{gathered}
$$

In order for Eq. (4.15) to constitute the selfexcitation referred to in Item (1), the coefficient of the 2nd term of Eq. (4.15) must have a nega-

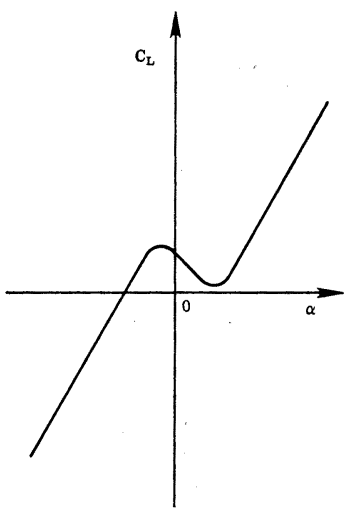

Fig. 16 An example of $\alpha-C_{L}$ curve tive value. Now, generally, $C$ and $C_{D}(0)$ are positive, so that a necessary condition for the above requirement would be $\left(\partial C_{L} / \partial \alpha\right)_{\alpha=0}<0$. Thus, violent vibration could be induced in the case indicated in Fig. 16, where, for flow attack on the nozzle at angle $\alpha=0$, the nozzle lift shows a negative slope. The present nozzle can be considered to present such a case.

(3) Measured negative damping

We derive in what follows the actual negative value of $\varepsilon$ in Eq. (4.3) from the measured data at the violent vibration given in Fig. 9 .

It was found that the difference of vibration amplitude was quite small for $\Delta t=T$, and so $\varepsilon$ was derived for $\Delta t=10 T$ :

From Eq. (4.5) with $T$ replaced by $10 T$, we have

$$
\varepsilon=-(1 / 10 T) \ln \left(\left|y_{t=t_{0}+10 T}\right| /\left|y_{t=t_{0}}\right|\right) .
$$

Substituting into Eq. (4.16)

$$
T=2 \pi / \nu_{d}, \quad \zeta=\varepsilon / \nu_{d},
$$

\begin{tabular}{|c|c|c|c|c|c|c|}
\hline 0 & 6 & 0.00800 & & 240 & 95 & 0.00622 \\
\hline 10 & 6.5 & 0.02683 & & 250 & 101.1 & 0.00520 \\
\hline 20 & 8.5 & 0.01112 & & 260 & 106.5 & 0.00223 \\
\hline 30 & 9.5 & 0.02336 & & 270 & 108.9 & 0.00528 \\
\hline 40 & 12 & 0.00408 & & 280 & 114.8 & 0.00384 \\
\hline 50 & 12.5 & -0.008338 & & 290 & 119.3 & 0.00394 \\
\hline 60 & 11.5 & 0.01226 & & 300 & 124.1 & 0.00379 \\
\hline 70 & 13 & 0.00377 & & 310 & 128.9 & 0.00177 \\
\hline 80 & 13.5 & 0.01054 & & 320 & 131.2 & 0.00204 \\
\hline 90 & 15 & 0.00953 & & $\mathbf{3 3 0}$ & 133.9 & 0.00445 \\
\hline 100 & 16.5 & 0.02647 & & 340 & 140 & 0.00420 \\
\hline 110 & 21.5 & 0.01706 & mean & 350 & 146 & 0.00449 \\
\hline 120 & 25.5 & 0.01790 & \rangle-0.01618 & 360 & 152.7 & 0.00227 \\
\hline 130 & 30.5 & 0.02458 & & 370 & 156.2 & 0.00272 \\
\hline 140 & 39 & 0.01138 & $\therefore \underline{\underline{s}}$ & 380 & 160.5 & 0.00136 \\
\hline 150 & 43.7 & 0.01347 & $=-0.0026$ & 390 & 162.7 & 0.00067 \\
\hline 160 & 50 & 0.01467 & & 400 & 163.8 & 0.00229 \\
\hline 170 & 57.9 & 0.00796 & & 410 & 167.6 & -0.00108 \\
\hline 180 & 62.7 & 0.00738 & & 420 & 165.8 & -0.00018 \\
\hline 190 & 67.5 & 0.00548 & & 430 & 165.5 & 0.00102 \\
\hline 200 & 71.3 & 0.00898 & & 440 & 167.2 & -0.00072 \\
\hline 210 & 78 & 0.00965 & & 450 & 166 & -0.00018 \\
\hline 220 & 85.9 & 0.00455 & & 460 & 165.3 & \\
\hline 230 & 89.9 & 0.00552 & & & & \\
\hline
\end{tabular}

where

$$
v_{d}=\sqrt{\nu^{2}-\varepsilon^{2}},
$$

we have the damping ratio

$$
\zeta=-(1 / 20 \pi) \ln \left(a_{n}+{ }_{10} / a_{n}\right) .
$$

where

$a_{n}$ : Double amplitude of data at $n$-th cycle

The actual values of $a_{n}$ corresponding to the measured data are presented in Table 3, from which a negative damping ratio was oftained as

$$
\zeta=-0.0026 \text {. }
$$

This means that the violent vibrations are based on the assumption of negative damping adopted in Sections 4.2.2. (1) and (2), though the

Table 3 Calculation of negative damping 
nozzle lift characteristics of nagative slope have not been verified.

\section{Measures Adopted for Preventing Violent Vibration}

The acceleration affecting the nozzles in question being of quite prominent intensity, as indicated from the data shown in Figs. 5, 6 and 7, it was felt that delay in implementing measures for abating the vibration risked serious damage. For this reason, measures to this end were immediately applied against both the causes considered probable in the preceding Chapter 4 , while the studies for further pinpointing the true cause were continued in parallel.

\subsection{For eliminating vibration due to Karman's vortex street}

Fins were mounted on the trailing edge of the nozzles, so as to decrease the trailing edge thickness from $100 \mathrm{~mm}$ to $16 \mathrm{~mm}$, hence to modity the vortex shedding frequency of the Karman's vortex streets, and thereby to aroid the resonance.

\subsection{For eliminating self-excited vibration due to negative damping}

The measure devised to eliminate vibration due to the mechanism discussed in Section 4.2.2 was as described below.

We simplify the expression of Eq. (4.15) by representing its 2 nd term in the form

$$
\zeta_{T}=\zeta_{S T}+\zeta_{S E V}
$$

where

$\zeta_{T}$ : Total damping ratio of nozzle

$\zeta_{s T}$ : Structural mass damping ratio of nozzle

$\zeta_{S E \text { I }}$ : Hydrodynamic damping ratio

We have seen in Section 4.2.2. (3) that

$$
\zeta_{T}=-0.0026 \text {. }
$$

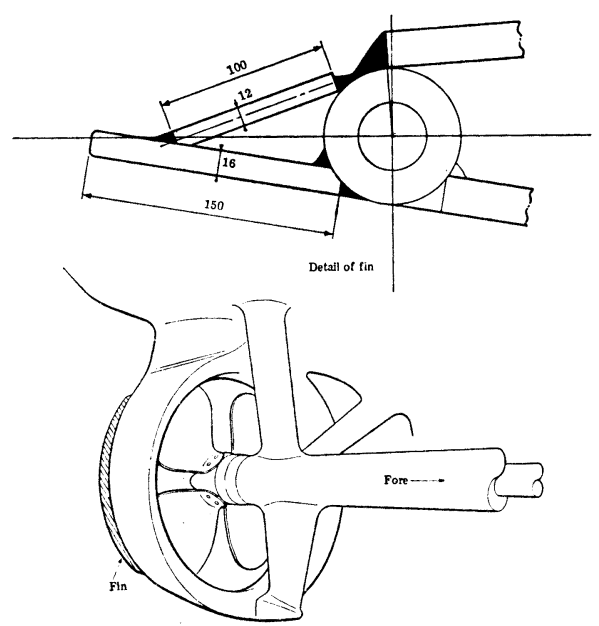

Fig. 17 Rough sketch of new fin
Self-excited vibration should be suppressible by rendering this value of $\zeta_{T}$ positive.

The results of external excitation applied to hull stern while moored ${ }^{1)}$ (Fig. 12) indicated that there was a marked difference in peak shape between the anti-phase and in-phase modes of vibration, which strongly suggested a large difference in damping ratio between the two modes of vibration. The actual values of structural and mass damping ratio estimated from measured data by Half Power Point method are.

$$
\begin{aligned}
\zeta_{S T}= & 0.0056 \text { for anti-phase } \\
& \text { vibration mode of nozzle } \\
\zeta_{S T}= & 0.0326 \text { for in-phase } \\
& \text { vibration mode of same },
\end{aligned}
$$

thus proving the damping ratio to be 6 times larger for the in-phase in comparison with antiphase mode.

Substituting into Eq. (5.1) the value for $\zeta_{S T}$ given by Eq. (5.2) and

$$
\zeta_{T}=-0.0026
$$

we have

$$
\zeta_{S E V}=-0.0082
$$

for anti-phase vibration mode of the nozzles. Assuming this hydrodynamic damping to remain unchanged and using $\zeta_{S T}$ in Eq. (5.3), we can calculate the total damping for in-phase mode as

$$
\begin{aligned}
\zeta_{T} & =0.0326-0.0082 \\
& =0.0244(>0) .
\end{aligned}
$$

This is resulting in a positive value of damping ratio, which should serve in eliminating the selfexcited vibration.

Thus, the in-phase mode of vibration was imposed on the nozzles by strutting them to each other in the arrangement illustrated in Fig. 18.

\subsection{Effectiveness of the measures}

After applying the measures vibration measurements were reperformed, again using underwater accelerometers, in order to verify the effectiveness of these measures. The measurements

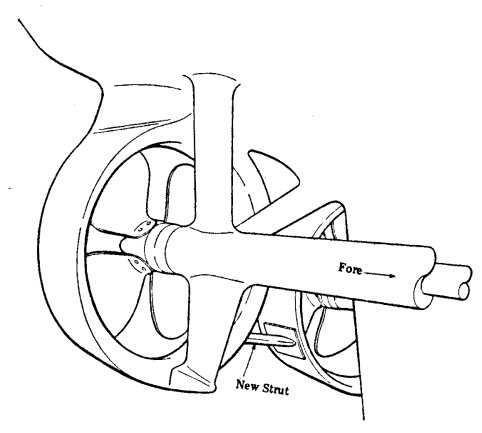

Fig. 18 Rough sketch of new strut 


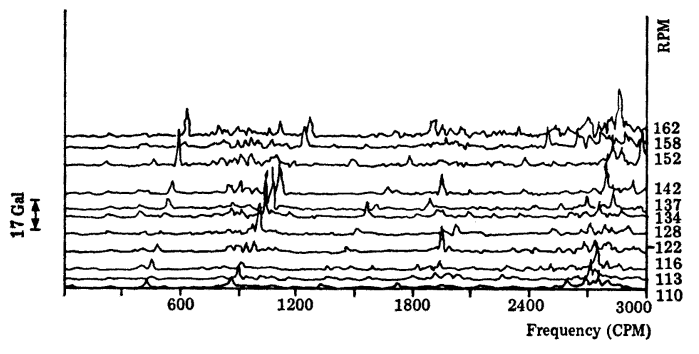

Fig. 19 An example of spectrum analysis V6(T) after measures were adopted

were conducted 4 times-twice on each tugboatwhich revealed complete suppression of all violent vibration such as observed in the previous Runs 1 and 3 .

As an example of the measured data, Fig. 19 reproduces the spectrum analysis of data from the measuring point V6, which had recorded the largest acceleration.

It is thus revealed that the self-excited vibrations that had affected the nozzles have been completely suppressed by the measures described in Section 5.5.

\section{Concluding Summary}

Two twin-engine twin-screw tugs built by IHI ranking amongst the world's largest-20,800 $\mathrm{SHP}, 200 \mathrm{tf}$ towing force, $19.4 \mathrm{kt}$ service speedand equipped with controllable-pitch propellers and fixed nozzles, were found after entrance into service to be affected by violent vibration of the nozzles.

The vibrations were measured and analyzed, from which it proved that:

(1) The vibrations occurred only in the range of propeller revolution between 113 and 140 rpm, and when the propeller blade pitch was at an angle exceeding $30^{\circ}$.

(2) The vibration frequency was invariably 826 $\mathrm{cpm}$ irrespective of propeller and engine revolution.

These facts pointed toward self-excited vibration induced by interaction between water flow and hull structure as cause of the violent vibrations, a phenomenon that had hardly ever been considered previously in ship vibration studies.

The two probable mechanisms considered as cause of this self-excited vibration were:

(1) Locked-in vibration induced by Karman's vortex streets occurring downstream of the nozzle trailing edges.

(2) Occurrence of negative damping.

Examination has so far proved neither of these probable mechanisms to be conclusively assignable as cause of the violent vibrations: (a)
Karman's vortex street was found upon calcular tion to provide excitation amplitude 2 orders of magnitude smaller than actually observed; the other surmise (b) of negative damping remains to be substantiated by definite tendency of the norzle lift characteristic presenting a negative slope against attack angle $\alpha$ around $0^{\circ}$.

Nonetheless, the vibrations observed were of such amplitude as to permit no time to be lost in investigations before taking remedial measures. For this reason, action was taken to counteract both the mechanisms considered probable: (a) Karman's vortex street by attaching fins to nozzle to modify the resonance velocity, and hence the exciting frequency, and (b) negative damping, by strutting between the two nozzles to modity the damping to positive value.

Vibration measurements reperformed after applying the above measures proved the violent vibrations to have been completely suppressed, evidencing ample effectiveness of the measures adopted.

Thus, while the immediate practical need of eliminating the violent vibrations affecting tugboat service operation has been satisfactorily solved, studies have been continued to investigate the true cause of the self-excited vibrations, as well as the pattern of flow past nozzle that induces the vibration. Other questions remaining to be answered include defining the range of operating conditions (propeller loading etc.) that should leave the nozzles free from vibration, and the elements of nozzle design that should contribute to avoiding vibration excitation. The results will be reported on the 2 nd Report.

In closing, the authors express their deep appreciation of the unreserved cooperation in according every facility for vibration measurements provided by the Owners of the two tugboats. Grateful acknowledgment is also due to Dr. Tadashi Matsudaira, now retired former IHI Research Institute Director, and Dr. Masaharu Kunieda, IHI Technical Advisor for their valuable advice and encouragement throughout the course of the present study.

\section{Reference}

1) Okumoto, Y., Yamazaki, K., Kumazaki, S.: Vibration of Ocean Tugboat IHI Engineering Review Vol. 14 No. 1 (Jan. 1981).

2) Japan Road Association: Specifications for heighway bridges (Feb. 1981).

3) Dr. Naudascher, E.: Flow-induced forces and vibrations Text book of Intensive Lectures on Flow-induced Vibrations, Tokyo, Japan (Sept. 1982). 